\title{
Application of Interferon Beta-1b in Multiple Sclerosis
}

\author{
Francesca Bagnato
}

Visiting Assistant Professor of Radiology and Radiological Sciences, Vanderbilt University Institute of Imaging Science

\begin{abstract}
Recombinant interferon beta-1b (IFN $\beta$-1b) was the first approved disease-modifying therapy for patients with multiple sclerosis (MS) by the world regulatory agencies for medical drugs and devices. IFN $\beta$-1b significantly decreases the inflammatory component of MS but still has doubtable effect on the neurodegenerative component. This article appraises the beneficial effects of IFN $\beta$ - $1 \mathrm{~b}$ on clinical and imaging measures of disease. Upon only briefly discussing the side effects of the drug, it will conclude with some crucial scientific aspects warranting urgent investigations to precisely address the potentials of IFN $\beta$-1b in MS patients.
\end{abstract}

\section{Keywords}

Multiple sclerosis, interferon beta-1 $\beta$ (IFN $\beta-1 \mathrm{~b})$, magnetic resonance imaging (MRI), inflammation, neurodegeneration

Disclosure: Francesca Bagnato acts as a consultant for reading and interpretation of scans in a six-month magnetic resonance imaging initiative project with Biogen Idec and has no other conflicts of interest to declare. The views and opinions expressed are solely those of the author in accordance with the reviewers' suggestions. Acknowledgements: The author thanks Professor Carlo Pozzilli (La Sapienza University of Rome) for critically revising the manuscript. The Intramural Research Program of the NINDS-NIH supported this research.

Received: 1 September 2010 Accepted: 31 January 2011 Citation: European Neurological Review, 2011;6(1):36-44 DOI:10.17925/ENR.2011.06.01.36 Correspondence: Francesca Bagnato, Department of Radiology and Radiological Sciences, Institute of Imaging Science, Vanderbilt University, 116121 st Avenue South, AA 1105 MCN, Nashville, TN 37232-2310, US. E: Francesca.r.bagnato@vanderbilt.edu

Multiple sclerosis (MS) is a chronic illness of the central nervous system affecting approximately 2.5 million young people worldwide. ${ }^{1}$ Regarded as exclusively a white matter (WM) disease, clinicians are now facing the notion, known to pathologists for over a century, that MS may affect the grey matter (GM) as well. ${ }^{2}$

Currently, MS is an incurable disease. It is, however, to some extent treatable. The past 20 years have witnessed a remarkable expansion of the horizons of pharmacological treatments aimed at delaying disease progression. Recombinant interferon beta-1b (IFN $\beta-1 \mathrm{~b}$ ) was the first disease-modifying therapy approved in MS by the US Food and Drug Administration (FDA). ${ }^{3}$ Today, IFN $\beta-1 \mathrm{~b}$ is the drug for which clinicians have the most experience with in chronically treating patients.

This article will attempt to appraise the status of IFN $\beta$-1b application in MS patients today. Upon reviewing the clinical-radiological presentation of the illness and its evolution over time, the effects of IFN $\beta-1 \mathrm{~b}$ on clinical and imaging measures of disease, as reported in the largest clinical trials, will be discussed. Then the side effects of the drug will briefly be covered. This review will conclude by addressing some crucial scientific aspects that are still open for investigation to estimate the actual potentials of IFN $\beta$ - $1 \mathrm{~b}$ treatment in MS patients.

Two types of IFN $\beta$ are currently available for MS treatment, namely IFN $\beta-1 a$ and IFN $\beta-1 b$. Their mechanisms of actions and clinical efficacy almost entirely overlap but, for the purpose of the present review, IFN $\beta-1 \mathrm{~b}$ will be focused on.

\section{Clinical-radiological Presentation and Evolution of Multiple Sclerosis}

The first clinically manifest event of MS is the relatively acute or subacute occurrence of one or more combined neurological symptoms, lasting at least 24 hours, in the absence of fever and infection, and suggestive of demyelination and inflammation. Such an event, namely clinical relapse, defines the status of clinically isolated syndrome (CIS). This is not considered clinically definite MS (CDMS) until a second attack occurs. Approximately $44 \%$ of CIS patients will remain free from a second attack within the following three years. ${ }^{4} \mathrm{Up}$ to $10 \%$ of CIS patients will not convert to CDMS over the course of the subsequent 20 years. ${ }^{5}$ Patients not converting to CDMS within the following three years tend to have fewer active lesions during the six months following the first attack (see Figure 1) than those who do convert to CDMS. ${ }^{4}$

In $85-90 \%$ of CDMS patients (MS hereafter) the disease starts and evolves with a relapsing-remitting (RR) course. RRMS is characterised by repeated and time-interleaved clinical relapses. These relapses may last from a few days to a few months and are sustained by inflammation directed against either the spinal cord or the brain tissue.

\section{Contrast-enhancing Lesions and Clinical Attacks}

Magnetic resonance imaging (MRI) mirrors inflammation by showing sharply demarcated focal WM contrast-enhancing lesions (CELS) in T1-weighted (T1-W) images obtained upon the injection of the contrast agent gadolinium-DTPA (see Figure 2A). CELS (brain CELS more than spinal cord ones) may be visible even in the absence of a 
clinically evident attack, with a clinical flare occurring on average with every $10 \mathrm{CELS}{ }^{6}$ Nevertheless, as seen for CIS patients as well as MS patients, the higher the number of clinically silent CELS over monthly MRIs, the higher the likelihood that a clinical attack will occur later on.?

CELS may have different evolution over time. Such an evolution likely depends upon the degree of myelin destruction and axonal degeneration or repair. This may all take place by the time of the first occurrence and continue to evolve upon enhancement resolution. The average life-span of CEL visibility on imaging is nearly one month, with almost all CELS terminating as hyperintense chronic lesions in T2-W images $^{8}$ (see Figure $2 B$ ). Nearly $20 \%$ of CELs culminate in persisting hypointensities in T1-W images ${ }^{9}$ (see Figure $2 C$ ).

T2-lesions have poor specificity with respect to the underlying pathological process and may represent areas with any degree of demyelination, axonal loss and repair. Conversely, T1-hypointense WM lesions persisting upon the enhancement resolution, namely persisting black holes (PBHs), are known to be pathologically more severe, since they are sustained by a higher degree of axonal loss. ${ }^{10}$ A subset of those PBHs appear as hyperintense lesions in cerebrospinal fluid tissue-specific imaging" (CSF-TSI, see Figure 2D). Lesions seen by CSF-TSI are likely a later event and are believed to represent cavities filled with CSF-like fluid as a final result of tissue death and loss.

As time progresses, the number of new clinical attacks and WM focal lesions is reduced. Some patients may still demonstrate a slow but constant accretion of physical, cognitive and emotional disability over time. Those patients are the ones shifting towards the secondary progressive (SP) phase of the disease. SPMS is predominantly characterized by WM and GM tissue loss and leads to irreversible clinical deterioration and neurodegeneration. As a result of a long-standing insidious neurodegenerative process, patients also tend to present with cortical thinning ${ }^{12}$ and focal cortical lesions, see Figures $2 E$ and $2 F^{13}$

The Role of Inflammation and Neurodegeneration In about $10-15 \%$ of patients, the disease starts and evolves with a primary progressive (PP) course. PPMS leads patients to disability accretion and rapid brain and spinal cord tissue volume loss, with little inflammation visible by MRI. ${ }^{14}$

Cumulatively, MS presents with two different components (inflammation and neurodegeneration) of variable duration over time within individual patients. An initial phase of inflammation ultimately fades into an ominous and occult neurodegenerative process that leads patients to irreversible disability. At any stage of the disease, the two components may overlap and may be regulated by a complex, yet unknown, interplay. The relationship between inflammation and neurodegeneration in MS is one of the most complicated in medical science and likely regulated by a number of genetic, immunological and environmental factors as yet unknown to clinicians and scientists.

Numerous drugs have been tested and proven to be effective in defeating or at least controlling the inflammatory phase of MS, with little or unknown independent effect on the neurodegenerative component. IFN $\beta-1 \mathrm{~b}$ belongs to this group of drugs.
Figure 1: Monthly Contrast-enhancing Lesion Activity in Clinically Isolated Syndrome Patients Converting and Non-converting to Multiple Sclerosis within Three Years

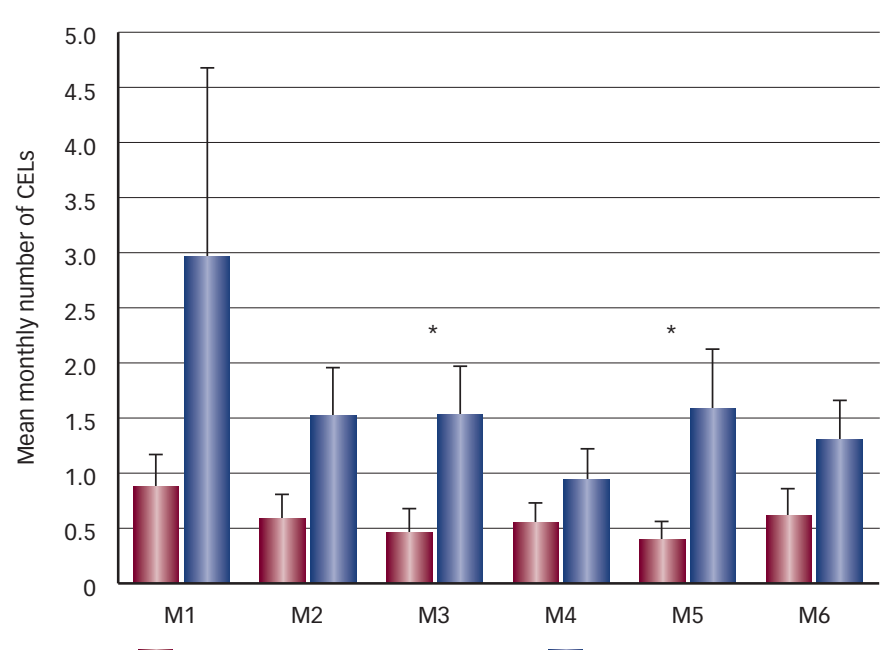

- 'Not converting to MS' $\mathrm{n}=32$

+ 'Converting to $\mathrm{MS}^{\prime} \mathrm{n}=32$

Although a clear trend towards higher contrast-enhancing lesions (CELS) number in clinically isolated syndrome (CIS) patients converting to multiple sclerosis is seen on a monthly basis, differences are statistically significant* at months three and five (by student $t$-tests). $M=$ month.

Data presented in this figure are courtesy of Professor Carlo Pozzili, La Sapienza University of Rome, Italy.

Figure 2: Active and Chronic Multiple Sclerosis Disease Visible by Magnetic Resonance Imaging

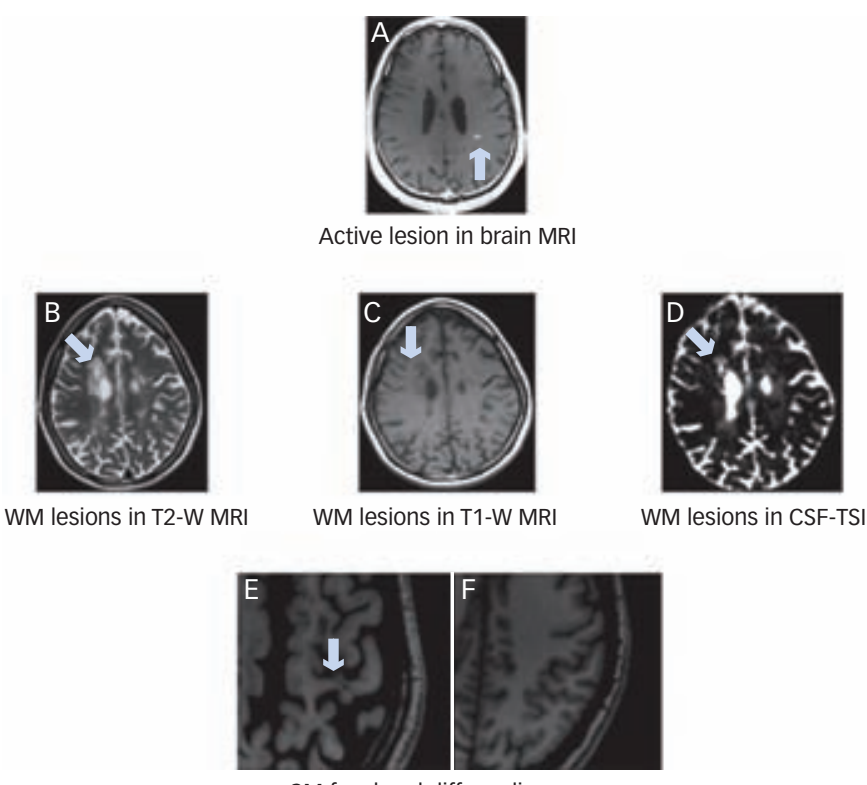

GM focal and diffuse disease

The arrow indicates an active brain contrast-enhancing lesion (A); in a patient with relapsing-remitting multiple sclerosis (RRMS), 7.5 years of MS and an Expanded Disability Status Scale (EDSS) score of 2.5. T2-weighted (T2-w) fast-spin echo (B); in a T1-w spin echo image (C); cerebrospinal fluid tissue specific imaging (CSF-TSI; D) showing persistent black hole (arrow in D) that is visible as in T2-w image (arrow in B) and as a CSF-TSI lesion (arrow in D). The image was acquired in a RRMS patient with an EDSS score of 6 and 33 years of disease. The T1-w inversion recovery spoiled gradient-recalled image of a 35-year-old secondary-progressive MS female patient with 18 years of disease and an EDSS score of 6 (E) and a 35-year-old healthy volunteer (F). The following three important details need to be derived from $E$ and $F$ : (1) an overall enlargement of the cerebrospinal fluid spaces at the expense of shrinking of the entire brain tissue is visible; (2) the magnetic resonance image (MRI) signal is reduced in the cortex of the patient (E), which also appears thinner compared with the cortex of the healthy volunteer (F); and (3) juxtacortical and cortical lesions are visible in the patient's MRI as indicated by the arrows. The MRIS presented in this figure were acquired using a 3Tesla GE scanner equipped with an eight-channel coil. $G M=$ grey matter; $W M=$ white matter

Images and clinical data are courtesy of Drs Jeff Duyn and Henry McFarland, National Institutes of Health, Bethesda, MD. Image reconstruction of CSF-TSI was facilitated by Dr Vasiliki Ikonomidou, George Mason University, Fairfax, VA. 


\section{Clinical and Imaging Parameters to Measure Disease Progression and Drug Efficacy in Multiple Sclerosis \\ Clinical Measures}

The main clinical metrics used thus far for measuring IFN $\beta$-1b's effect in MS are:

- relapse rate;

- proportion of relapse-free patients;

- severity of relapse;

- disability progression according to the Expanded Disability Status Scale (EDSS); ${ }^{15}$

- proportion of patients free from EDSS change; and

- change in MS functional composite (MSFC) score. ${ }^{16}$

The meaning of clinical relapses was clarified earlier in this article. With respect to severity, the following measures are considered indicative of disease severity.

- extent of functional recovery;

- need to use steroid medication and/or hospitalise the patient; and

- number of days of hospitalisation.

Numerous clinical scales are used to measure physical, cognitive and emotional disability of MS patients. Nevertheless, the ones commonly employed thus far are the EDSS and the MSFC scale.

The EDSS scale ranges from 0 to 10 with 0.5 -step increases and quantifies MS-induced disability in the pyramidal, cerebellar, brainstem, sensory, bowel, bladder, visual and cerebral functional systems. EDSS steps 1 to 4.5 are indicative of MS patients who have independent walking ability. EDSS steps 5.0 to 9.5 are defined by severe impairment to ambulation. A sustained progression is defined as any change $\geq 1$ for EDSS scores $\leq 5$ and any change $\geq 0.5$ for EDSS scores $\leq 5.5$. The variables most commonly used to identify drug effects are: the proportion of patients who remain free from disability progression over three- or six-month time-windows; time to sustained disability progression; group- and/or within-person differences over time in median EDSS scores.

The MSFC is a 3D scale that measures cognitive function, leg function/ambulation, arm/hand motor and co-ordination functions. The scale produces values that are translated into z-scores in reference to normative values. These scores are used for group comparisons or for within-person comparison over a set time period.

\section{Imaging Measures}

The use of MRI provides a number of measurable disease parameters by means of which disease progression can be monitored. This is achieved with the use of conventional and non-conventional MRI techniques. ${ }^{17}$ Conventional MRI techniques include T1-W and T2-W spin echo images. They permit the identification and computation of acute and chronic WM lesions (see details later). These measures are currently mostly employed in clinical trials. WM lesion identification and computation is a sensitive method for assessing disease progression and at the same time is highly reproducible across different centres. Non-conventional MRI techniques allow one to obtain quantitative measurements of brain and spinal cord normal-appearing WM (NAWM), normal-appearing GM and lesions. one such technique is magnetisation transfer imaging (MTI)
Quantities derived from MTI, namely magnetisation transfer ratios (MTRS), are considered to indirectly reflect the amount of demyelination and axonal loss within visible lesions as well as in regions of normal-appearing tissue and lesions.

Compared with conventional MRI techniques, non-conventional ones offer several challenges when one attempts their application in large multicentre clinical trials. First, scanning time may considerably increase. Second, results may suffer inter-centre variability, therefore being poorly reproducible. Third, sophisticated image post-processing may be required for some of the non-conventional techniques, rendering their application in large clinical trials unpractical. To date, besides limited applications, which will be discussed later in the course of this review, no studies have attempted to investigate the effects of IFN $\beta$-1b on MRI quantities derived from measurements on normal-appearing tissue and lesions.

A third image technique that is considered a non-conventional MRI technique but reflects the application of advanced post-processing analysis in conventional sequences, is the measurement of brain volume and its changes over time. Such a measurement is achieved by quantifying the so-called brain parenchyma fraction (BPF). This is given by the ratio of the sum of the WM and GM volumes over the volume of the entire brain, i.e. $(\mathrm{WM}+\mathrm{GM}) /(\mathrm{WM}+\mathrm{GM}+\mathrm{CSF})$. The concept behind the application of BPF is that as the ventricles enlarge due to brain tissue shrinkage, the BPF decreases. BPF measurements have been successfully applied in large clinical trials. ${ }^{18}$

\section{Types of Study Design}

Different types of study design have been used in MS to detect the effect of IFN $\beta-1 b$. Study type depends upon two main factors:

- the number of subjects needed to treat in order to detect a significant effect on the chosen primary outcome measure; and

- ethical motivations that over time limit the possibility of assigning patients to the placebo control arm.

Two main types of study designs have been used to identify the effect of IFN $\beta-1 \mathrm{~b}$ in MS thus far: randomised controlled trials (RCTs) and baseline versus therapy (BVT) studies.

\section{Radomised Controlled Trials}

In RCTs patients are randomly allocated to the 'intervention' or placebo group and both investigators and patients are blind with respect to the compounds administered. This type of study design provides important data regarding the efficacy of IFN $\beta$ - $1 \mathrm{~b}$ compared with placebo in reducing the number and severity of clinical relapses as well as newly-formed lesions on MRI.

RCTs led to the approval of IFN $\beta-1 b$ by regulatory agencies for medical drugs and devices worldwide. They were subsequently slightly modified by substituting the placebo with another approved disease-modifying therapy. This type of 'active comparator' study allows the superiority of IFN $\beta$ - 1 b versus other standard-of-care or experimental compounds to be measured.

\section{Baseline versus Therapy Studies}

A BVT study is where each patient receives exposure to the drug after a natural history evaluation period. The main advantages offered by this type of study versus the RCTs are: 
- each patient serves as his/her own control, thus minimising the effect of inter-patient variability in measuring drug effect; and

- it generally requires a smaller number of subjects.

However, at the same time BVT studies eliminate the blinding component of the RCT, thus potentially introducing the so-called Pygmalion type of bias. In MS, studies of this led to the initial identification of IFN $\beta$ - 1 b's efficacy in decreasing the number of active lesions or CELS within individual patients.

Presently, in patients with MS, placebo-controlled trials can still ethically be prepared, but with restrictions. Specifically, for MS patients for whom established effective therapies exist, placebocontrolled trials should only be offered with rigorous informed consent if the patients refuse any of the existing therapies, have not responded to them, or if these treatments are not available for other reasons, such as cost. ${ }^{19}$

\section{Interferon Beta-1b Effects on Clinical and Imaging Disease Parameters}

This section discusses the effects of IFN $\beta$ - $1 \mathrm{~b}$ on clinical and imaging measures of disease seen in the main clinical trials. Clinical and imaging findings will be presented separately. For each type of finding, evidence obtained in each patient subgroup, i.e. CIS, RRMS and SPMS with and without superimposed relapses and PPMS, is discussed.

In the majority of clinical trials and post-marketing studies, IFN $\beta$-1b has been administered at a dosage of 8 million international units, equivalent to $250 \mu g$, subcutaneously every other day (EOD). For the purpose of this review, this dosage is always referred to as the as standard dose unless otherwise indicated. In addition, when specifying that the effects of the drug were found to be significant, a $p$-value $\leq 0.05$ is always referred to. Readers are advised to consult the individual papers referred to for additional details regarding the magnitude of alpha error of each study.

\section{Clinical Measures}

\section{Frequency and Severity of Clinical Relapses}

When looking at differences in clinical relapse rate between patients treated with IFN $\beta-1 \mathrm{~b}$ and patients in the placebo group, a statistically significant reduction was seen and maintained over the course of two-year observational periods in patients with $\mathrm{CIS}^{20}{ }^{20} \mathrm{RMMS}^{21}$ and SPMS with superimposed relapses. ${ }^{22}$ On average, one in every seven RRMS ${ }^{21}$ or one in every nine $\mathrm{CIS}^{20}$ patients will remain relapse-free for two years if treated with $250 \mu \mathrm{g}$ IFN $\beta-1 \mathrm{~b}$. It is important to highlight that when a greater proportion of CIS patients remain relapse-free, ultimately a greater proportion have a delay in time to conversion to $\mathrm{MS}^{20}$

Such reductions, to some extent dose-dependent, ${ }^{21}$ are attributable to an average two-fold decrease in the frequency of moderate and severe attacks in treated versus untreated patients. Subcutaneous

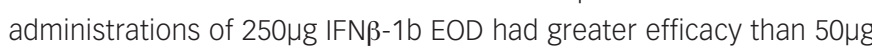
EOD. ${ }^{21}$ On clinical relapse, $250 \mu \mathrm{I}$ IFN $\beta$-1b was not inferior to:

- $500 \mu g$ subcutaneous IFN $\beta-1 b$ EOD; 23

- $22 \mu \mathrm{g}$ IFN $\beta-1 a$ subcutaneously once weekly:24

- $44 \mu \mathrm{g}$ IFN $\beta$-1a subcutaneously three times per week; or ${ }^{25}$

- $20 \mathrm{mg}$ subcutaneous glatimerar acetate (GA) daily ${ }^{23}$ or EOD. ${ }^{26}$

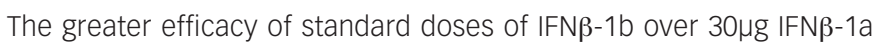
given intramuscularly once weekly has been proven. ${ }^{25,27}$ In SPMS patients without superimposed relapses, IFN $3-1 \mathrm{~b} 250 \mu \mathrm{g}$ or $160 \mu \mathrm{g} / \mathrm{m}^{2}$ body surface area administered EOD significantly decreased the number of clinical attacks over a time period of up to two years. ${ }^{28}$

Observational periods longer than two years have provided controversial results. In CIS patients, early treatment with $250 \mu \mathrm{g}$ IFN $\beta$-1b maintained a reduced annualised relapse rate for only one additional year, but not from year four of therapy onwards versus patients starting therapy two years later. ${ }^{29}$ In RRMS patients, a one-third reduction in exacerbation rate was maintained for up to five years versus placebo. ${ }^{30}$ Differences in mean yearly exacerbation rate between patients treated with a standard dose of IFN $\beta-1 \mathrm{~b}$ and those

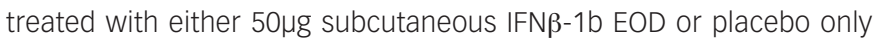
remained statistically significant until the end of the second year of therapy..$^{30}$ Conversely, a lower rate of severe versus moderate relapses was seen throughout five years in the standard-dose group. ${ }^{30}$ In SPMS patients with superimposed relapses, a statistically relevant decrease in mean relapse rate was sustained for up to four years in treated patients. ${ }^{31}$

\section{Disability Progression}

When one looks at the effect of a given drug in reducing disability progression, two important considerations need be kept in mind. First, disability changes during therapy might be linked to the indirect effect of a given drug in reducing the number and severity of clinical relapses, thereby leading to slower or lower accumulation of disability over time. Second, changes in disability might be due to a direct effect of the drug in promoting faster and more efficient remyelination or relenting demyelination. This will favor slower and less pronounced disability accumulation independently from the effect of reducing clinical relapse.

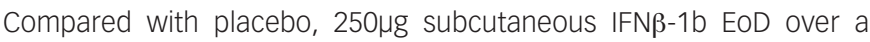
two-year time period significantly reduced the proportion of patients experiencing a sustained progression in disability among those with CIS, RRMS and SPMS with superimposed relapses..$^{20,21,22}$ As seen with relapse rate, 250 $\mathrm{\mu g}$ was superior to $50 \mathrm{\mu g}$ administered EOD ${ }^{21}$ but was not inferior to the following subcutaneous regimens for remaining free from disability progression or having relevant beneficial changes

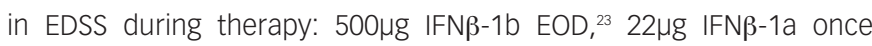
weekly ${ }^{24}$ and $44 \mu \mathrm{g}$ IFN $\beta$-1a three times per week. ${ }^{25}$ Interestingly, on measures of disability progression by EDSS scores, 250 $\mathrm{\mu g}$ subcutaneous IFN $\beta-1 \mathrm{~b}$ EOD was superior to 20mg subuctaneous GA daily ${ }^{23}$ or EOD ${ }^{26}$ and $30 \mu \mathrm{ug}$ intramuscular IFN $\beta$-1a weekly. ${ }^{24,27}$ None of the effects of IFN $\beta-1 b$ on physical disability were seen in patients with SPMS without superimposed relapses ${ }^{28}$ or in those with PPMS. ${ }^{32}$ It is important to highlight that although IFN $\beta$-1 $\mathrm{b}$ keeps a higher number of patients free from disability progression, it does not necessarily imply that the EDSS scores of treated versus untreated patients or patients before and after therapy are statistically different.

Few studies have looked at the effect of IFN $\beta$-1b on MSFC score. Over a two-year observational period, a significant improvement in MSFC score was seen in patients with PPMS treated with IFN $\beta-1 b$ compared with those taking placebo. ${ }^{32}$ This improvement was not maintained beyond three months after drug discontinuation. Nor was MSFC score paralleled by improvement in other cognitive tests evaluating verbal fluency and processing speed. Conversely, in 
Figure 3: Contrast-enhancing Lesion Decrease During Therapy with Interferon Beta-1b
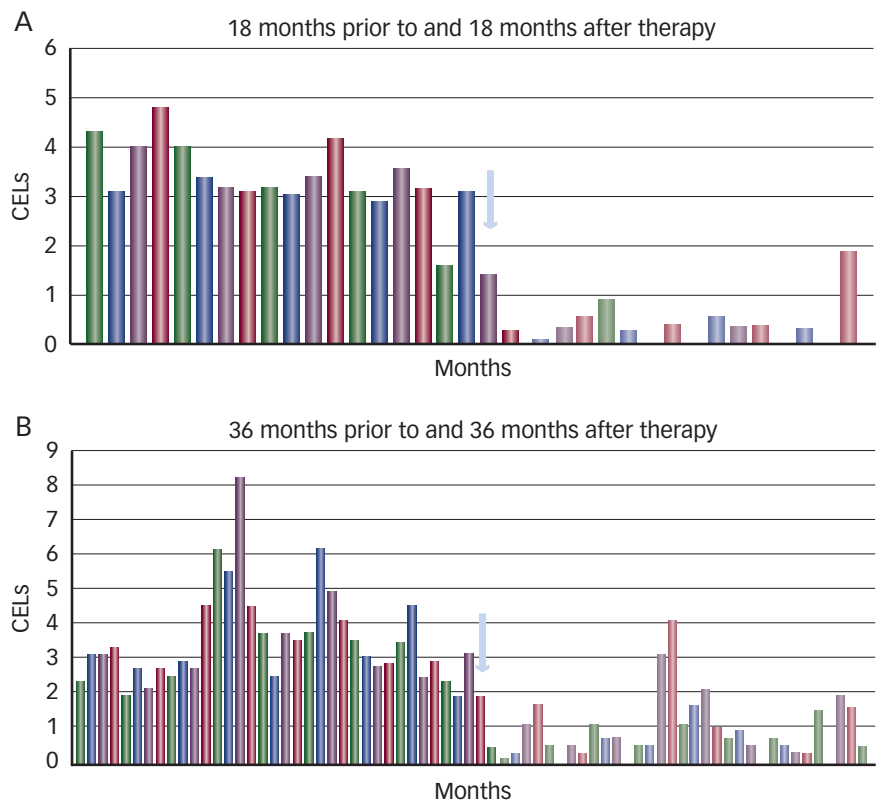

A: Mean monthly contrast-enhancing lesion (CEL) activity 18 months before and 18 months after therapy with interferon beta-1b (IFN $\beta$-1b) in a cohort of 10 patients with multiple sclerosis (MS). The drug significantly reduced the mean number of CELS:40 B: Mean monthly CEL activity 36 months before and 36 months after therapy with IFN $\beta-1 b$ in a cohort of six patients with MS. The drug significantly reduced the mean number of CELS. ${ }^{41}$ The arrow indicates therapy start.

patients with CIS a non-significant MSFC score improvement was seen between early- and late-treated patients after five years. ${ }^{29}$ Interestingly, such improvement was driven by the cognitive component of MSFC score that, more than any other component, may be affected by patient training. ${ }^{33}$

In an attempt to understand the long-term effect of IFN $\beta$-1b in MS patients (i.e. beyond five years) following completion of the pivotal trial, 372 RRMS participants were contacted by physicians and enrolled in a 16-year survey. Of the 372 patients initially enrolled, 260 (69.9\% of the original group) had EDSS evaluations after 16 years of follow-up. These patients were taking various medications, as recommended by their physician. Although there is no statistical analysis in this long term follow-up, descriptive results indicate that

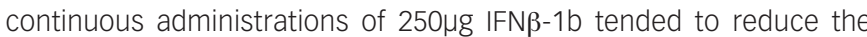
proportion of patients converting to SPMS and progressing to an EDSS

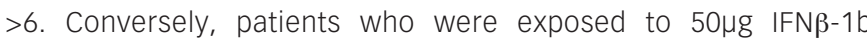
progressed faster and more frequently to SPMS and were more likely to end up with an EDSS score $>6 .{ }^{34}$

\section{Imaging Measures}

Magnetic Resonance Imaging Measures of Active Inflammation In addressing the question of whether IFN $\beta$-1b has an effect on blood-brain barrier (BBB) breakdown and inflammation, one should consider two important pathogenetic points. First, it is crucial to investigate and assess whether IFN $\beta$-1b or any other given drug can prevent BBB breakdown. such an assessment permits the anti-inflammatory and immunomodulating property of a given drug to be established. Second, it is important to examine the ability of IFN $\beta$-1b to halt the WM changes preceding CEL occurrence before BBB breakdown and, later, promoting faster and better CEL recovery. The latter would ultimately provide evidence of the indirect effects of IFN $\beta-1 \mathrm{~b}$ as neuroprotective agent.
With respect to the effect of IFN $\beta-1 \mathrm{~b}$ before BBB breakdown, two main imaging outcome measures have been used thus far, namely number of CELS in T1-W post-contrast MRI and number of newly-formed lesions in T2-w images. From a statistical stand-point, defining new lesions as either active T2-lesions or CELS as MR end-point does not seem to be substantially different for monitoring MRI activity in MS clinical trials. ${ }^{35}$ However, biologically the advantage of CELS over newly-formed T2-lesions is given by the fact that CELS directly visualise the BBB breakdown while newly-formed T2-lesions may be only an indirect harbinger of an ongoing inflammatory process or inflammation that has occured previously.

The effect of IFN $\beta-1 b$ on CELS has not been investigated in all clinical trials, mainly due to the cost of studying this. Monitoring CEL activity not only adds the costs of the contrast agent itself to the study, but also requires numerous monthly scans that certainly further increase costs. The effect of IFN $\beta-1 \mathrm{~b}$ beyond BBB resolution and quality of inflammatory activity has only been assessed sporadically. This has been carried out by obtaining pre-therapy versus therapy-phase but also treated versus untreated patient comparisons in:

- the proportion of CELS converting into PBHS;

- the size of CELS formed during the different pre-therapy and therapy-phases; and

- the evolution of CEL by MTR measurements.

\section{Interferon Beta-1b Effect on Lesion Quantity}

That IFN $\beta-1 \mathrm{~b}$ reduces the number of CELS, newly-formed and growing T2-lesions over a two-year time period has been extensively demonstrated in large RCTs performed in patients with $\mathrm{CIS}_{1}{ }^{21} \mathrm{RRMS}_{1}{ }^{21,23,24,27,36}$ SPMS with ${ }^{22}$ and without superimposed relapse ${ }^{28}$ and PPMS.$^{32}$ Such an effect was preserved on a yearly basis for up to four years in CIS patients, ${ }^{29}$ five years in RRMS patients ${ }^{30}$

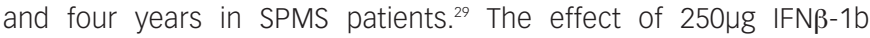
administered subcutaneously EOD on T2-lesions was similar to

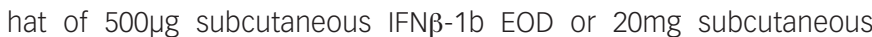
GA daily. ${ }^{23}$

Using both a fixed and a random effect model to investigate the distribution of active T2-lesion reduction over a three-year follow-up of SPMS patients with superimposed relapses, it has consistently been shown that between-patient response to IFN $\beta$-1b in T2-lesion reduction is highly heterogeneous. ${ }^{37}$ Reduction in T2 lesions account for only $57 \%$ of the of the treatment's effect on disability progression and $68 \%$ of the effect on relapse rate reduction. ${ }^{38}$ Patients receiving treatment had $>65 \%$ chance of showing $\geq 60 \%$ T2-lesion reduction but also a $7 \%$ probability that there would be no reduction or even an increase in T2-lesion size. ${ }^{37}$

When looking at the results of the small open-label BVT studies performed thus far, IFN $\beta$ - $1 \mathrm{~b}$ administrations significantly decreased the number of new and total CELs for up to 19 months of follow-up. ${ }^{39}$ Similarly, a significant overall reduction in monthly CEL frequency was detected during therapy phases of $18^{40}$ (see Figure $3 A$ ) and 36 months $^{41}$ (see Figure $3 B$ ) in comparison to a natural history phase of equal time.

As seen for the distribution of active T2-lesion reduction in SPMS, individual variability and month-to-month fluctuations in drug effects were also noted in these small studies, which relied upon CEL counting. ${ }^{42}$ Specifically, $\geq 60 \%$ reduction in CEL activity was achieved 
and remained constant over three years in slightly more than $50 \%$ of patients. An additional $13.7 \%$ of individuals had a $60 \%$ reduction in CEL activity after less significant changes initially. The remaining patients never reached $\geq 60 \%$ reduction in CEL frequency.

\section{Interferon Beta-1b Effect on Contrast-enhancing Lesion Quality}

To evaluate the effect of IFN $\beta-1 \mathrm{~b}$ on CEL quality, several imaging measures were considered. The first such measure was the proportion of CELS evolving into PBHs. Studies performed by the National Institutes of Neurological Disorders and Stroke (NINDS) group on RRMS patients yielded contradictory findings depending on the group of patients studied. In a 72-month open-label BVT study performed on six patients with RRMS, patients were observed to have a significantly reduced likelihood of forming PBHs from CELS forming during therapy than during the pre-therapy phase..11 No similar significant effect was observed in a shorter 36-month follow-up study including $10 \mathrm{MS}$ patients (eight RRMS and two with SPMS). ${ }^{43}$

The ability of IFN $\beta-1 \mathrm{~b}$ to reduce the likelihood of PBH formation from CELS in RRMS has recently been confirmed by Filippi and co-authors in a large RCT whereby patients were assigned to receive either IFN $\beta-1 \mathrm{~b}$ or $\mathrm{GA} .{ }^{44}$ The drugs were found to be equally significantly effective in protecting patients against $\mathrm{PBH}$ formation during therapy. Conversely, when patients with SPMS and superimposed relapses were examined, Brex and co-authors failed to find any significant decrease in the proportion of CELS converting into PBHS 18 months later compared with placebo. ${ }^{45} \mathrm{~A}$ more extensive and detailed description of IFN $\beta-1 b$ reducing the likelihood of CEL progression to $\mathrm{PBH}$ has been reported by the NINDS group. ${ }^{46}$

A second imaging marker used to identify the effect of IFN $\beta$-1 1 b on CEL quality is CEL size. The size of a given CEL is known to be an indirect indicator of the underlying disease pathology. Larger CELS have a greater tendency to evolve into PBHs, have lower MTRs and tend to be present in SPMS more than in RRMS patients. A relatively large study performed in the NINDS group during a short-term (six-month) time window, has shown that the effect of IFN $\beta$-1b in reducing the likelihood of CEL formation is not paralleled by its ability to reduce the size of CELS once formed. ${ }^{47}$ IFN $\beta$-1b reduced the number of CELs by $76.4 \%$ over 18 months of therapy. This decrease however, was statistically greater for small lesions enhancing only once during therapy (82.3\%) than for larger ones enhancing multiple times $(57.4 \%){ }^{40}$

A third imaging measure used to reliably track CEL evolution over time and investigate the ability of IFN $\beta$ - $1 \mathrm{~b}$ to promote faster recovery is monthly measurements of MTR. The effect in CEL MTR is rather limited. As early as 12 months before the occurrence of enhancement, MTR starts to decrease. Such a pre-lesional decrease in MTR is not affected by IFN $\beta$ - $1 \mathrm{~b}$. In addition, the extent of CEL MTR recovery over time appears to be more evident if steroids are used in association with IFN $\beta-1 \mathrm{~b} .{ }^{48}$

\section{Magnetic Resonance Imaging Measures of Neurodegeneration} In addressing the question of whether IFN $\beta-1 \mathrm{~b}$ has an effect on imaging measures of neurodeneration, two important measures need to be considered. First, one should keep in mind measures of focal chronic lesions, such as lesion volume (LV) in T1-w (i.e PBHs) and T2-W images. Second, one should consider the effect of IFN $\beta-1 \mathrm{~b}$ in measures of global brain and spinal cord degeneration. These measures include brain volume loss (or BPF changes), whole-brain MTR, NAWM-MTR and cervical cord area measurements at the level of the second cervical vertebra or $\mathrm{C} 2$.

\section{Interferon Beta-1b Effect on Focal Chronic Lesions}

The role of IFN $\beta-1 \mathrm{~b}$ in reducing T2-LV is accompanied by a dual component. First, changes in T2-LV may reflect changes in chronic T2-lesions present prior to therapy. Second, those changes may only reflect the effect of the drug on newly-formed lesions. In general, as

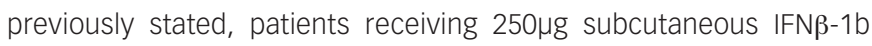
EOD tend to form fewer new lesions (as seen in T2-w images). As a result, the difference in total T2-LV between treated and untreated patients at the end of the study may reach statistical significance. Such results were seen in patients with $\mathrm{CIS}_{1}{ }^{20,29} \mathrm{RRMS}^{23,30,36}$ SPMS with $^{22,31}$ and without $^{28}$ superimposed relapses and in those with PPMS. ${ }^{32}$ Interestingly, in patients with CIS and RRMS, group differences remained significant throughout years four and five, respectively. As stated earlier, however, these results do not necessarily imply that IFN $\beta$-1b affects, by decreasing their size, the number of chronic lesions in T2-W images present at the time of the therapy start. Similarly, when one looks at the LV of PBHs, there are no reports to date that have sought to determine the role of IFN $\beta-1 b$ in preventing the formation of new PBHs or in decreasing the quantity of pre-existing PBHs, separately.

Kappos and co-authors measured the volume of PBHs in CIS patients on therapy with IFN $\beta$ - $1 \mathrm{~b}$ for up to five years and failed to demonstrate a drug effect. ${ }^{29}$ Conversely, Barkhof and co-authors measured the PBH volume changes over a three-year observation period in a subgroup of 95 patients with SPMS and superimposed relapses and found that the IFN $\beta$-1b-treated group had a significantly slower accumulation of PBHS at 36 months. ${ }^{49}$ These results were confirmed by Montalban and co-authors, who performed the same type of analysis in a cohort PPMS patients treated for 24 months with the same therapy regimen..$^{32}$ $\mathrm{O}^{\prime}$ Connor and co-authors found the same in RRMS patients. ${ }^{23}$ With respect to the latter study, it is interesting to highlight that $250 \mu \mathrm{g}$ subcutaneous IFN $\beta$-1b EOD had a superior effect on T2-lesions but not on T1-lesions compared with $20 \mathrm{mg}$ subcutaneous GA administered daily. ${ }^{23}$ In previous open-label BVT observational studies performed at the the National Institutes of Health, a decrease in the number of PBHS forming de novo during therapy has been shown. Nevertheless, the longevity of PBHs arising during the natural history phase is no shorter than those formed during therapy. Consequently, according to these findings the ability of IFN $\beta-1 b$ to promote the formation of less aggressive CELS or a faster recovery over time should be questioned..$^{41}$

\section{Interferon Beta-1b Effect on Global Disease - Brain Parenchyma Fraction and Magnetisation Transfer Ratios}

The reduction of brain atrophy accumulation over time with IFN $\beta-1 b$ is controversial. At variable observational time periods, no significant differences in loss of brain volume were seen in treated versus untreated CIS (five years), ${ }^{29}$ SPMS with superimposed relapses (three years) ${ }^{50}$ or PPMS (two years). ${ }^{32}$ Conversely, IFN $\beta$ - $1 \mathrm{~b}$ seemed to protect the brain from atrophy better than GA in patients with RRMS. ${ }^{23}$ Similarly, over a three-year time period, an open-label study conducted in RRMS patients showed that IFN $\beta-1 b$ successfully decreased brain volume loss with respect to pre-therapy phase. ${ }^{51}$ 
Figure 4: Relation Between Contrast-enhancing Lesions Decrease and Atrophy Increase in a Treated Multiple Sclerosis Patient
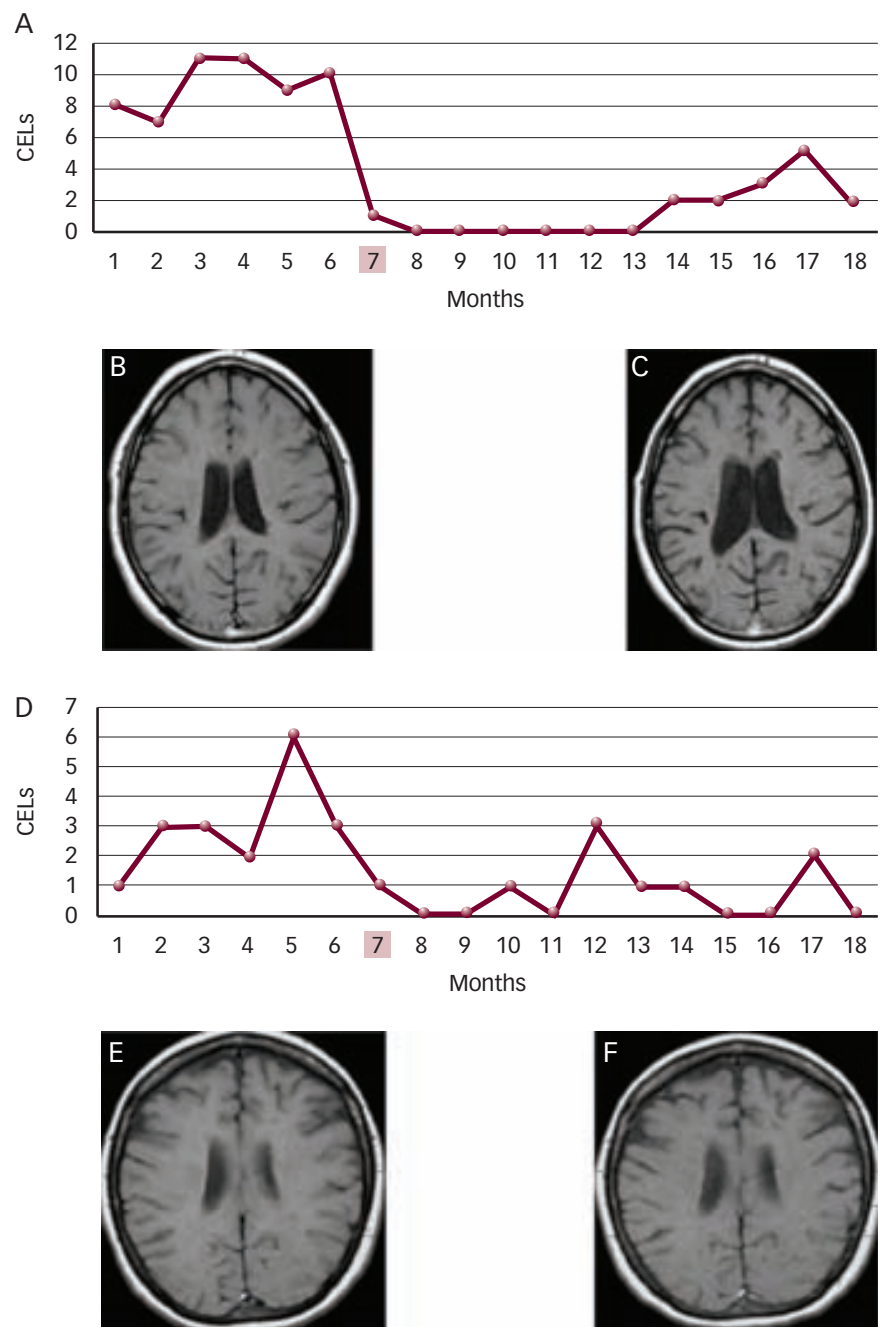

The chart represents monthly contrast-enhancing lesions (CELS) in a responder (A) and non-responder (D) patient. The red box in the chart indicates the start of therapy. The responder is defined on the basis of a monthly reduction by $\geq 60 \%$ in the number of CELS during the first 12 months of therapy compared with the six-month pre-therapy phase. For the same patients, baseline (B-C) and 10-year (E-F) T1-weighted (T1-W) spin echo magnetic resonance images are shown. One can see relatively larger ventricle enlargement in the responder patient by $C E L$ of $A-C$, whose annualised rate of brain atrophy decrease was $0.55 \%$ compared with the non-responder patient by CEL in D-F, whose brain atrophy annualised rate of decrease was 0.33\% (Bagnato F, unpublished data).

The magnetic resonance images presented in this figure were acquired using a 1.5Tesla GE scanner equipped with a standard head coil.

The magnetic resonance images presented in this figure are courtesy of Joseph Frank and Henry MCFarland, National Institutes of Health, Bethesda, MD. Image post-processing was facilitated MS Jailan Hanafy and Carolyne Bavan, NIH, Bethesda, MD.

As seen with BPF, no significant effect of the whole brain and NAWM regions was seen to be exerted in MTR by IFN $\beta$-1b in SPMS patients with superimposed relapses ${ }^{52}$ or PPMS ${ }^{32}$ after three and two years of therapy, respectively. Finally, no significant changes in spinal cord area measurements were seen to be exerted by IFN $\beta-1 b$ in treated versus untreated patients over the course of two years of therapy. ${ }^{32}$

The effect of IFN $\beta-1 \mathrm{~b}$ in reducing neurodegeneration, as measured by brain atrophy, is still being debated and is controversial. When visible, such an effect has been shown to be dependent on two main factors: time and the CEL-status of the patient. With respect to time, it is hypothesised that in the short term, IFN $\beta$-1b may induce accelerated, non-tissue-related brain volume loss (i.e. pseudoatrophy) due to the reduction of CELS and inflammation-related oedema. ${ }^{18,50}$ The actual biological substrate of the effect of IFN $\beta-1 \mathrm{~b}$ in reducing brain atrophy accumulation may become visible later on, once the oedema is resolved.

With respect to CEL patient status, controversies and uncertainties still dominate. Exploratory subgroup analyses of the SPMS cohort of treated patients indicated that patients without CELS prior to therapy had a significantly greater reduction in cerebral volume in the placebo group compared with those with CELS prior to therapy. There was a trend towards greater reduction in cerebral volume, however, if the patient was on therapy. Although those differences did not reach a statistically relevant threshold, the trend suggests that in untreated patients no relation exists between inflammation and neurodegeneration. Conversely, when patients are taking IFN $\beta-1 \mathrm{~b}$, the reduction in inflammatory activity is paralleled by halting of brain volume loss. Similarly, monthly MRI analyses of individual RRMS cases performed in the NINDS group have shown that a decrease in the accumulation of brain atrophy is generally associated with decreases in the number of CELS. ${ }^{53}$

The fundamental question unanswered by all these studies is to what extent reduction in brain atrophy accumulation is merely sustained by CEL reduction or whether there is an additional effect independently exerted by IFN $\beta-1$ b. To this end, two patients who exemplify the complexity of the relationship between inflammation and neurodegneration when IFN $\beta-1 \mathrm{~b}$ is active are discussed here (see Figure 4). Both patients were monitored monthly with six MRIs prior therapy and 12 MRIs during the first 12 months of therapy and one MRI at year 10 of therapy. The patient represented in Figure $4 A-C$ had a substantial ( $\leq 60 \%$ decrease) monthly decrease in CEL number, but his atrophy progressed at a rate of $-0.55 \%$ yearly. Such a progression rate is similar to that observed in untreated MS patients. Over the 10-year follow-up period, he shifted from RRMS the SPMS with an EDSS increase of 3.5 (from 2.5 to 6.0). Conversely, the patient in Figure $4 D-F$ was not an optimal CEL responder during the first year of therapy but her atrophy progressed at a rate of $-0.33 \%$ per year, which is similar to what is observed in healthy subjects. Over the 10-year treatment period, her disease remained RRMS and she had an EDSS increase of 0.5 (from 1 to 1.5).

\section{Interferon Beta-1b Side Effects, Tolerability and Resistance}

IFN $\beta-1 \mathrm{~b}$ is a relatively well-tolerated drug. Its side effects are generally manageable with temporary drug discontinuation, although discontinuation is rarely necessary due to side-effects. The most common side effects include lymphopenia, injection-site reaction, flu-like syndrome, headache, pain and thyroid dysfunctions.

Very few patients exhibit depression and suicidal ideas. Compared with IFN $\alpha$ which circulates for longer than IFN $\beta-1 b$ in the blood, depression does not occur de novo in patients treated with IFN $\beta-1 \mathrm{~b}$. Despite this, care needs to be taken in those patients with depressive symptoms prior to therapy, since these patients may exhibit suicidal ideas as therapy starts. This topic is only briefly expanded upon since numerous extensive reviews and post-marketing studies have been compiled on the topic..$^{54,55,56}$ Specifically, it is recommended that readers consult an important long-term (16-year) follow-up study on the safety and tolerability of IFN $\beta-1 \mathrm{~b}$ very recently published ${ }^{57}$ and several informative reports on the effect of IFN $\beta$ - 1 b on the quality of life of MS patients. ${ }^{58,59,60}$ Today there is class III evidence that RRMS 


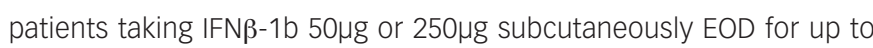
five years, with subsequent unspecified treatment, have fewer deaths after 16 years of follow-up than similar patients on placebo for up to five years, with subsequent unspecified treatment. An important problem associated with therapy with IFN $\beta-1 \mathrm{~b}$ is the development of neutralising antibodies (NABS). NABS may occur after as little as six months of therapy and persist for a few years. Whether NABs are the cause or epiphenomenon of therapy failure is still a controversial question that, similarly to the topic of side effects, has been extensively debated in the literature. ${ }^{.1,62,63}$

\section{Conclusions}

IFN $\beta-1 \mathrm{~b}$ is one of the most widely used and effective therapies for preventing disease progression in MS. Despite this, several uncertainties remain in the clinical community regarding the actual therapeutic potential of IFN $\beta$-1b. Undoubtedly, the combined effort of basic and clinical scientists is warranted to provide insights into the several still-unanswered questions.

\section{Interferon Beta-1b Beside Blood-Brain Barrier Breakdown}

Both imaging and clinical evidence thus far prove that when the inflammatory component of MS still plays the major visible pathogenetic role, IFN $\beta$ - $1 \mathrm{~b}$ seems to have a key effect in preventing the occurrence of new flares. However, the effect of IFN $\beta-1 b$ on neurodegeneration and disability accretion remains controversial. In addition, it is unclear whether the effect of IFN $\beta-1 \mathrm{~b}$ in halting neurodegeneration progress is independent of its indirect effect on inflammation or is an epiphenomenon. Studies designed to elucidate this topic have never been conducted and are certainly warranted.

\section{Interferon Beta-1b Before Blood-Brain Barrier Breakdown and Beyond its Resolution}

Limited and discouraging evidence is available on the (in)ability of IFN $\beta-1 b$ to affect NAWM pre-lesional or outside-lesion MRI quantities. Further knowledge on such topic would ultimately allow one to understand:
- to what extent IFN $\beta$-1b alters the disease course before CELS become visible; and

- the need to treat patients without visible CELS.

Serial imaging studies in large patient cohorts examining the effect of IFN $\beta-1 \mathrm{~b}$ on the changes occurring in NAWM before a lesion develops and once its enhancement resolves are crucial.

\section{Interferon Beta-1b and Grey-matter Disease}

No evidence exists thus far on the role of IFN $\beta-1 \mathrm{~b}$ in affecting focal and diffuse GM disease in MS. Lack of such knowledge impedes elucidation of the neuroprotective properties of the drug. Nowadays numerous quantitative and non-quantitative MRI techniques are available to identify part of the cortical lesions and topographically measure selective damage to the GM. Using these techniques in the context of clinical trials is the next step.

\section{Interferon Beta-1b Beyond Clinical Relapses and Physical Disability}

Although the effect of IFN $\beta-1 \mathrm{~b}$ in decreasing the occurrence of new clinical relapses and relenting physical disability progression is established, no evidence is available on its ability to halt other disease-associated symptoms. Among these symptoms, cognitive and emotional disability play a substantial role in affecting the life of MS patients. Exploring the interplay between IFN $\beta-1 \mathrm{~b}$ and these symptoms is hence crucial in order to define the actual potential of the drug.

Current knowledge yields the conclusion that in patients with MS, IFN $\beta-1 \mathrm{~b}$ partially affects the quantity of disease but probably leaves its quality unaltered. Conversely, quality of disease leads patients to irreversible disability as much as, if not more than, its quantity.

The combined effort of clinicians and basic scientists is required to disentangle this topic, which will ultimately permit understanding the role of IFN $\beta-1 b$, if any, in the complex interplay between inflammation and neurodegeneration in MS
1. Ramagopalan SV, Dobson R, Meier UC, et al., Multiple sclerosis: risk factors, prodromes, and potential causa pathways, Lancet Neurol, 2010;9:727-39.

2. Lassmann $H$, The pathologic substrate of magnetic resonance alterations in multiple sclerosis, Neuroimaging Clin N Am, 2008;18:563-76.

3. Nightingale SL, From the Food and Drug Administration, JAMA, 1993;270:1672.

4. Tomassini V, De Giglio L, Reindl M, et al., Anti-myelin antibodies predict the clinical outcome after a first episode suggestive of MS, Mult Scler, 2007:13:1086-94.

5. Fisniku LK, Brex PA, Altmann DR, et al., Disability and T2 MR lesions: a 20-year follow-up of patients with relapse onset of multiple sclerosis, Brain, 2008;131:808-17.

6. McFarland HF, Frank JA, Albert PS, et al., Using gadolinium-enhanced magnetic resonance imaging lesions to monitor the activity in multiple sclerosis, Ann Neurol, 1992;32:758-66.

7. Kappos L, Moeri D, Radue EW, et al., Predictive value of gadolinium-enhanced magnetic resonance imaging for relapse rate and changes in disability or impairment in multiple sclerosis: a meta-analysis. Gadolinium MRI multiple sclerosis. a meta-analysis. Gadolinium

8. Thompson AJ, Kermode AG, Wicks D, et al. Thompson AJ, Kermode AG, Wicks D, et al.,
Major differences in the dynamics of primary and secondary progressive multiple sclerosis, Ann Neurol 1991;29:53-62.

9. Bagnato F, Jeffries N, Richert ND, et al., Evolution of T1 black holes in patients with multiple sclerosis imaged monthly for 4 years, Brain, 2003;126:1782-9.

10. Van Waesberghe $\mathrm{JH}$, Kamphorst W, De Groot CJ, et al ، Axonal loss in multiple sclerosis lesions: magnetic Axonal loss in multiple sclerosis lesions: magnetic
resonance imaging insights into substrates of disability, resonance imaging insights

11. Riva M, Ikonomidou VN, Ostuni JJ, et al., Tissue-specific imaging is a robust methodology to differentiate in vivo t1 black holes with advanced multiple sclerosis-induced damage, AJNR, 2009;30:1394-401.

12. Sailer M, Fischl B, Salat $D$, et al., Focal thinning of the cerebral cortex in multiple sclerosis, Brain 2003; 126:1734-44

13. Bagnato F, Butman J, Gupta S, et al., Cortical plaques in patients with multiple sclerosis: in vivo MRI study, AJNR, 2006;27:2161-7

14. Confavreux C, vukusik $S$, Natural history of multiple sclerosis: a unifying concept, Brain, 2006:129:606-16.

5. Kurtzke JF, Rating neurologic impairment in multiple sclerosis: an expanded disability status scale (EDSS), sclerosis: an expanded disabit

16. Cutter GR, Baier ML, Rudick RA, et al., Development of a multiple sclerosis functional composite as a clinical trial outcome measure, Brain, 1999;122:871-82.

17. Bagnato $F$, Frank JA, The role of nonconventional magnetic resonance imaging techniques in demyelinating disorders, Curr Neurol Neurosci Rep, 2003;3:238-45.

18. Miller DH, Barkhof F, Frank JA, et al., Measurement of atrophy in multiple sclerosis: pathological basis, methodological aspects and clinical relevance, Brain, methodological aspect

19. Polman $\mathrm{CH}$, Reingold $\mathrm{SC}$, Barkhof $\mathrm{F}$, et al., Ethics of placebo-controlled clinical trials in multiple sclerosis: a reassessment, Neurology, 2008;70:1134-40.

20. Kappos L, Polman CH, Freedman MS, et al., Treatment with interferon beta-1 $\mathrm{b}$ delays conversion to clinically definite and $\mathrm{MCD}$ onald $\mathrm{MS}$ in patients with clinically isolated syndrome, Neurology, 2006;67:1242-9.

21. The IFNB Multiple Sclerosis Study Group, Interferon beta-1b is effective in relapsing-remitting multiple sclerosis. Clinical results of a multicenter, randomized, double-blind, placebo-controlled trial, Neurology, 1993;43:655-61.
22. Kappos L, European Study Group on interferon beta-1b in secondary progressive MS, Placebo-controlled multicentre randomised trial of interferon beta- $1 \mathrm{~b}$ in treatment of secondary progressive multiple sclerosis, Lancet 1998; 7:352:1491-7.

23. O' Connor P, Arnason B, Comi G, et al., 250 microg or 500 microg interferon beta- $1 \mathrm{~b}$ versus $20 \mathrm{mg}$ glatiramer acetate in relapsing-remitting multiple sclerosis: a prospective, randomised, multicentre study, Lancet Neurol, 2009:8:889-97.

24. Koch-Henriksen N, Sorensen PS, Christsensen T, et al., A randomized study of two interferon-beta treatments in relapsing-remitting multiple sclerosis, Neurology, 2006;66:1056-60.

25. Etemadifar $M$, Janghorbani $M$, Shaygannejad V, Comparison of Betaferon, Avonex, and Rebif in treatment of relapsing-remitting multiple sclerosis, Acta Neurol scand, 2006;113:283-7.

26. Fletcher S, Vardi J, Pollak L, et al., Comparison of glatimerar acetate (Copaxone) and interferon beta-1b (Betaferon) in multiple sclerosis patients: an open-label 2-year follow-up I Neurol Sci, 2002;197:51-5.

27. Durelli L, Verdun E, Barbero P, et al., Every-other-day interferon beta-1 $1 \mathrm{~b}$ versus once-weekly interferon beta-1a for multiple sclerosis: results of a 2-year prospective randomised multicentre study (INCOMIN), Lancet, 2002;359:1453-60

28. Panitch $H$, Miller $A$, Paty $D$, et al., Interferon beta- $1 \mathrm{~b}$ in secondary progressive MS: results from a 3-year controlled study, Neurology, 2004;63:1788-95

29. Kappos L, Freedman MS, Polman $\mathrm{CH}$, et al., Long-term effect of early treatment with interferon beta- $1 \mathrm{~b}$ after a first clinical event suggestive of multiple sclerosis: 5 -year active treatment extension of the phase 3 BENEFIT trial, Lancet treatment extension of the

30. The IFNB Multiple Sclerosis Study group and the University 
of British Columbia MS/MRI Analysis Group, Interferon beta- $1 \mathrm{~b}$ in the treatment of multiple sclerosis; final outcome of the randomized controlled trial, Neurology, 1995;45:1277-85

31. Kappos L, Polman C, Pozzilli C, et al, Final analysis of the European multicenter trial on IFN beta-1 $\mathrm{b}$ in secondary-progressive MS, Neurology, 2001;57:1969-75.

32. Montalban $X$, Sastre-Garriga J, Tintoré $M$, et al. $A$ single-center, randomized, double-blind, placebo-controlled study of interferon beta-1 $\mathrm{b}$ on primary progressive an transitional multiple sclerosis, Mult Scler, 2009;15:1195-205.

33. Solari A, Radice D, Manneschi L, et al., The multiple sclerosis functional composite: different practice effects in the three test components, J Neurol Sci, 2005;228:71-4.

34. Ebers GC, Traboulsee A, Li D, et al., Analysis of clinical outcomes according to original treatment groups 16 years after the pivotal IFNB-1b trial, I Neurol Nuerosurg Psychiatry, 2010;81(8):907-12.

35. Bonzano L, Roccatagliata L, Mancardi GL, et al , Gadolinium-enhancing or active T2 magnetic resonance imaging lesions in multiple sclerosis clinical trials? Mult Scler, 2009;15:1043-7.

36. Paty DW, Li DK, The UBC MS/MRI Study Group, the IFNB Multiple Sclerosis Study Group, Interferon beta-1b is effective in relapsing remitting multiple sclerosis. II. MRI analysis results of a multicenter, randomized, double-blind, placebo-controlled trial, Neurology, 1993:43:662-7.

37. Sormani MP, Bruzzi P, Beckmann $\mathrm{K}$, et al., MRI metrics as surrogate endpoints for EDSS progression in SPMS patients treated with IFN beta-1b, Neurology, 2003;60:1462-6.

38. Sormani MP, Bruzzi P, Beckmann K, et al., The distributio of magnetic resonance imaging response to interferon beta-1b in multiple sclerosis, J Neurol, 2005;252:1455-8.

39. Stone LA, Frank JA, Albert PS, et al., The effect of interferon-beta on blood-brain barrier disruptions demonstrated by contrast-enhanced magnetic resonance imaging in relapsing-remitting multiple sclerosis, Ann Neurol, 1995:37:611-9.

40. Gupta S, Solomon J, Tasciyan T, et al., Interferon beta effects on re-enhancing lesions in patients with multiple sclerosis, Mult scler, 2005;11:658-68.
41. Bagnato F, Gupta S, Richert ND, et al., Effects of interferon beta-1b on black holes in multiple sclerosis over a 6-year period with monthly evaluations, Arch Neuro 2005;62:1684-8

42. Chiu A, Richert N, Ehrmantraut M, et al., Heterogeneity in the imaging response patterns to interferon beta in patients with multiple sclerosis: a 3 -year study with monthly imaging Arch Neurol, 2009:66:39-43.

43. Bagnato $F$, Jeffries $N$, Richert $N$, et al., Interferon beta therapy does not affect duration in time of Gd-enhancin lesions and black holes in relapsing remitting multiple sclerosis [Abstract], American Academy of Neurology, 55th Annual Meeting, Honolulu, HI, 2003.

44. Filippi M, Rocca MA, Camesasca F, Interferon beta-1b compared with GA therapy for reducing evolution of permanent T1 hypointensities (black holes) on brain MRI in permanent Tre hypoin Academy of Neurology, 62th Annual Meeting, Toronto, Canada, April 2010

45. Brex PA, Molyneux PD, Smiddy P, et al., The effect of IFNbeta-1 $\mathrm{b}$ on the evolution of enhancing lesions in secondary progressive MS, Neurology, 2001;57:2185-90.

46. Bagnato F, Evangelou IE, Gallo A, et al., The effect of interferon-beta on black holes in patients with multiple sclerosis, Expert Opin Biol Ther, 2007:7:1079-91.

47. Gaindh $\mathrm{D}$, Jeffries N, Richert N, et al., The role of interferon beta $1 \mathrm{~b}$ in decreasing lesions size in patients with multiple sclerosis, Expert Opin Biol Ther, 2008:12:1823-9.

48. Richert ND, Ostuni JL, Bash CN, et al., Interferon beta-1b and intravenous methylprednisolone promote lesion recovery in multiple sclerosis, Mult scler, 2001;7:49-58. 9. Barkhof F, Van Waesberghe JH, Filippi M, et al., $T(1)$ hypointense lesions in secondary progressive multiple sclerosis: effect of interferon beta-1b treatment, Brain, 2001; 124:1396-402.

50. Molyneux PD, Kappos L, Polman C, et al., The effect of interferon beta- $1 \mathrm{~b}$ treatment on MRI measures of cerebra atrophy in secondary progressive multiple sclerosis. European Study Group on Interferon beta-1b in secondary progressive multiple sclerosis, Brain, 2000;123:2256-63.

51. Frank JA, Richert N, Bash C, et al., Interferon-beta-1b slows progression of atrophy in RRMS: Three-year follow-up in NAb- and NAb+ patients, Neurology, 2004;62:719-25.

52. Inglese $\mathrm{M}$, van Waesberghe $\mathrm{JH}$, Rovaris $\mathrm{M}$, et al., The effect of interferon beta-1b on quantities derived from MT MRI in secondary progressive MS, Neurology, 2003;60:853-60.

53. Richert ND, Howard T, Frank JA, et al., Relationship between inflammatory atrophy and cerebral atrophy in multiple sclerosis, Neurology, 2006;66:551-6.

54. Galetta SL, Markowitz C, US FDA-approve disease-modifying treatments for multiple sclerosis: review of adverse effect profiles, CNS Drugs, 2005;19:239-52.

55. Lam S, Wang S, Gottesman M, Interferon-beta1b for the treatment of multiple sclerosis, Expert Opin Drug Metab Toxicol, 2008:4:1111-7

56. Paolicelli D, Direnzo V, Trojano M, Review of interferon beta- $1 \mathrm{~b}$ in the treatment of early and relapsing multiple sclerosis, Biologics, 2009:3:369-76.

57. Reder AT, Ebers GC, Traboulsee A, et al., Cross-sectional study assessing long-term safety of interferon-beta-1b for relapsing-remitting MS, Neurology, 2010;74:1877-85.

58. Schwartz CE, Coulthard-Morris L, Cole B, et al., The quality-of-life effects of interferon beta-1b in multiple sclerosis. An extended Q-TWiST analysis, Arch Neurol, 1997;54:1475-80.

59. Rice GP, Oger J, Duquette $P$, et al., Treatment with interferon beta-1b improves quality of life in multiple sclerosis, Can I Neurol SCi, 1999:26:276-82.

60. Lily $\mathrm{O}$, McFadden $\mathrm{E}$, Hensor $\mathrm{E}$, et al., Disease-specific quality of life in multiple sclerosis: the effect of disease modifying treatment, Mult Scler, 2006;12:808-13.

61. Bagnato $F$, Riva $M$, Antonelli $G$, The role of neutralizing antibodies in patients with multiple sclerosis, Expert Opin Biol Ther, 2006;6:773-85.

62. Deisenhammer F, Neutralizing antibodies to interferon-beta and other immunological treatments for multiple sclerosis: prevalence and impact on outcomes, CNS Drugs, 2009;23:379-96.

63. Polman $\mathrm{CH}$, Bertolotto $\mathrm{A}$, Deisenhammer $\mathrm{F}$, et al., Recommendations for clinical use of data on neutralising antibodies to interferon-beta therapy in multiple sclerosis, Lancet Neurol, 2010;9:740-50. 